\title{
Using a membrane-penetrating-peptide to anchor ligand in the liposome membrane facilitates targeted drug delivery
}

Xiaobo Fan ${ }^{\dagger *}$, Hongbo Xu ${ }^{\dagger}$, Junlong Song ${ }^{\ddagger}$, Yongcan Jin ${ }^{\ddagger}$, Michael Wink ${ }^{\S}$, Guoqiu Wu ${ }^{\# *}$

$\dagger$ Diagnostics Department, Medical School, Southeast University, Nanjing 21009, China. Email:101011951@seu.edu.cn. Tel\&Fax:+86-25-83272357

$\dagger$ Jiangsu Provincial Key Laboratory of Pulp and Paper Science \& Technology, Nanjing Forestry University, Nanjing 210009, China.

§ Institute of Pharmacy and Molecular Biology, Heidelberg University, Heidelberg 69120, Germany

\# Center for Clinical Laboratory Medicine of Zhongda Hospital, Southeast University, Nanjing 21009, China. E-mail:101008404@seu.edu.cn. Tel\&Fax:+86-25-83272355

*Corresponding to: X Fan, E-mail:101011951@seu.edu.cn. Tel: +86-25-83272357; G Wu, Email:101008404@seu.edu.cn. Tel: +86-25-83272355 


\title{
Methods and materials
}

\author{
Strains, plasmids and cell lines
}

E. coli Rosetta DE3 cells and the plasmid pET28a were purchased from Merck KGaA (Darmstadt, Germany) and used for protein expression. The DNA of the targeting fusion protein IGP (Genbank accession no.MK 572728) were manually designed and the expression plasmids were ordered from Shanghai Generain Co., Ltd. (Shanghai, China; order no. PO01848). Standard strains from the American Type Culture Collection (ATCC) were provided by Zhongda Hospital, Southeast University. Adenocarcinomic human alveolar basal epithelial A549 cells and human embryonic kidney 293 cells (HEK293) were purchased from Institute of Basic Medical Sciences, Chinese Academy of Medical Sciences (Beijing, China). Thanatin, Ib-AMP4 and P1c were ordered from Scipeptide Co., Ltd. (Shanghai, China) (Supporting information Table S1\&Fig.S9).

\section{Medium and other materials}

Tryptone and Ni-NTA affinity columns were purchased from GE Health (GE Healthcare Biosciences, Pittsburgh, USA). Dulbecco's Modified Eagle's Medium (DMEM), Roswell Park Memorial Institute 1640 (RPMI 1640) medium and bovine serum were purchased from Gibco (Shanghai, China) and used for cell culture. Modified Luria-Bertani (LB) medium containing extra $5 \mathrm{~g} / \mathrm{L}$ calcium chloride was used for protein expression. Salt-free dextrose tryptone broth (DTB) containing $10 \mathrm{~g} / \mathrm{L}$ tryptone and $5 \mathrm{~g} / \mathrm{L}$ glucose was used for antimicrobial assays. Chloramphenicol, kanamycin and glucose were purchased from Sigma-Aldrich (Munich, Germany). Polyethyleneglycol (2000)-carbamyl distearoylphosphatidylethanolamine (DSPE-PEG2000$\mathrm{COOH})$, DOPC (1,2-dioleoyl-sn-glycero-3-phosphocholine) and DOPG (1,2-dioleoyl-sn-glycero3-phospho-(1'-rac-glycerol)) from Avanti (Avanti Polar Lipids, Alabaster, Alabama) were dissolved in chloroform/methanol $(9: 1)$ at $2.5 \mathrm{mg} / \mathrm{mL}$ to generate stock solutions. 1-(3dimethylaminopropyl)-3-ethyl-carbodiimide (EDC) and N-Hydroxysulfosuccinimide sodium salt (Sulfo-NHS) were obtained from Sigma (St. Louis, MO, USA). 3-(4,5-dimethyl-2-thiazolyl)-2,5diphenyl-2-H-tetrazolium bromide (MTT) was purchased from Aladdin (Aladdin Industrial Corporation, Shanghai, China) Other chemicals used in this study were of analytical grade or higher.

\section{Expression and purification of fusion proteins in E. coli BL21}

In this study, Ib-AMP4 were expressed as fusion with the reporter green fluorescent protein (GFP) (hereafter abbreviated as IGP). The expression and purification of fusion proteins were carried out as previously reported ${ }^{1}$. The fusion proteins were purified by Ni-NTA affinity chromatography, followed by desalting through dialysis against $5 \%$ glucose. The desalted proteins were utilized in subsequent experiments. The purity and content of the proteins were characterized by SDS-PAGE after each step.

\section{Vesicle preparation}

Small unilamellar liposomes were prepared by the lipid film rehydration method for QCM-D, ITC and TEM experiments. A DOPC:DOPG (3:1) lipid film mimicking the cellular membrane was dried from a chloroform/methanol solution under a nitrogen stream, followed by $3 \mathrm{~h}$ of vacuum drying. The dry film was subsequently rehydrated with 5\% glucose/PBS buffer ( $\mathrm{pH} 7.4)$ and then extruded 21 times through a polycarbonate membrane with $200 \mathrm{~nm}$ pores. IGP-decorated liposomes were prepared by the same method but were rehydrated with 5\% glucose containing IGP proteins. 
The chemical conjugation was performed as previously described ${ }^{2}$. In brief, liposomes were prepared from DOPC:DOPG lipid mixture supplemented with 5 mol \% DSPE-PEG-COOH by the lipid film hydration method followed by homogenization. Chemicals NHS/EDC were next supplied and incubated for $4 \mathrm{~h}$ at $4{ }^{\circ} \mathrm{C}$ to activate the carboxyl group of DSPE-PEG-COOH. IGP proteins and BSA were then added in turn to link with the DSPE-PEG-COOH. Chemicals and free proteins required to be removed after the preparation.

The doxorubicin (DOX) loaded DOPC/DOPG liposomes were prepared by ammonium sulfate gradient dialysis. A lipid film prepared from $4 \mathrm{mg}$ DOPC/DOPG was rehydrated with $1 \mathrm{~mL}$ of 250 $\mathrm{mM}$ ammonium sulfate solution followed by extrusion of 21 times through a polycarbonate membrane with $200 \mathrm{~nm}$ pores. The liposome preparation was then dialysed against PBS for $2 \mathrm{~h}$, and then incubated at $37^{\circ} \mathrm{C}$ for $1 \mathrm{~h}$ after addition of $1 \mathrm{mg}$ DOX dissolved in $1 \mathrm{~mL}$ of $5 \%$ glucose solution. The free DOX was removed by dialysis (300 kDa cut-off) against PBS for $2 \mathrm{~h}$.

The RBC liposomes was prepared after lysis. Approximately $10^{\wedge} 9 \mathrm{RBCs}$ were repeatedly washed with pure water until the hemoglobin was completely removed. RBC pellets resuspended in PBS were ultrasonicated at $300 \mathrm{~W}$ for 10 60 min and were then extruded 21 times through a polycarbonate membrane with $200-\mathrm{nm}$ pores for homogenization. IGP decorated RBC liposomes were prepared by pre-incubating the RBC pellets in 5\% glucose solution containing IGP proteins for 10 min before ultrasonication and extrusion. DOX encapsulated RBC liposomes were prepared by ammonium sulfate gradient dialysis. The RBC pellet was resuspended in $250 \mathrm{mM}$ ammonium sulfate solution followed by ultrasonication and extrusion to prepare empty RBC liposomes. The empty liposomes were next subjected to dialysis against PBS. Afterwards, $1 \mathrm{mg} / \mathrm{mL}$ DOX was supplemented and the liposomes were incubated at $60{ }^{\circ} \mathrm{C}$ for $20 \mathrm{~min}$. The cyanine 5 (Cy5)encapsulated liposomes were prepared by adding $1 \mathrm{mg} / \mathrm{mL}$ Cy 5 into the $\mathrm{RBC}$ pellets with or without IGP decoration followed by ultrasonication and extrusion. The free DOX, IGP and Cy5 was removed by dialysis (molecular weight (MW) cut-off $300 \mathrm{kDa}$ ) against PBS.

\section{Characterization of Liposomes}

The mean size, polydispersity index (PDI) and zeta potential of the prepared liposomes were determined using a Zetasizer Nano ZS ZEN3600 instrument (Malvern Instruments Ltd., Worchestershire, UK). The drug release rates of DOX loaded liposomes were measured at different pHs. A volume of $10 \mathrm{~mL}$ liposomes was dialysed (MW cut-off $300 \mathrm{kDa}$ ) against PBS at different pHs. An aliquot was withdrawn at each interval followed by ultrasonicated at $300 \mathrm{~W}$ for $10 \mathrm{~min}$. The DOX concentration was determined by a fluorescent spectrometer (FluoroMax-4, Horiba, Kyoto, Japan). The concentrations of the IGP proteins decorated on the liposomes were determined by the GFP fluorescence intensity after dialyisis (MW cut-off $300 \mathrm{kDa}$ ) against PBS.

\section{Quartz crystal microbalance with dissipation (QCM-D)}

The interaction between IGP protein and the DOPC-DOPG bilayer was evaluated using a QCM-D E4 system (Q-Sense, Gothenburg, Sweden) with thermostat control at $25^{\circ} \mathrm{C}$. Overtones of $5^{\text {th }}, 7^{\text {th }}$ and $9^{\text {th }}$ were recorded. Before liposomes were applied, the QCM-D quartz was characterized with a standard resonance frequency of $\sim 5 \mathrm{MHz}$. After liposome adsorption was complete, the quartz was rinsed with buffer and then normalized to zero before being loaded with different concentrations of IGP protein in 5\% glucose solution, followed by washes with PBS. The interaction between IGP and the lipid membrane was reflected by a shift in frequency and energy dissipation. To eliminate 
nonspecific binding, BSA was added to the glucose buffer during the rising and binding steps.

\section{Isothermal titration calorimetry experiments}

IGP protein and DOPC-DOPG liposomes were synchronously dialyzed against $5 \%$ glucose prior to use in experiments. The liposomes were placed in the reaction cell titrated against $4.1 \mathrm{mg} / \mathrm{mL}$ IGP protein in the syringe, and the reference cell was filled with deionized water. IGP was sequentially added in $2 \mu \mathrm{L}$ aliquots at $2 \mathrm{~min}$ intervals for a total of 21 injections. The molecular ratio of IGP:DOPC/DOPG shifted from 0:100 to 4:100 during ITC experiments. The data were processed using MicroCal Origin software. The titration data were deconvoluted based on a binding model containing "One set of sites" using a nonlinear least-squares algorithm.

\section{Cell culture and MTT assay}

A549 and HEK293 cells were used as $\alpha v \beta 3$-expression-positive and $\alpha v \beta 3$-expression-negative groups, respectively. The cells were cultured in DMEM medium containing $10 \%$ foetal bovine serum (FBS), $100 \mathrm{U} / \mathrm{mL}$ penicillin and $0.1 \mathrm{mg} / \mathrm{mL}$ streptomycin at $37^{\circ} \mathrm{C}$ in a humidified atmosphere containing $5 \% \mathrm{CO}_{2}$. The cell viability after exposure to different treatments was evaluated by MTT assay.

\section{Confocal laser scanning microscopy (CLSM) and flow cytometry}

IGP rupturing RBCs was monitored under CLSM. The purified RBCs were incubated for $10 \mathrm{~min}$ at $4{ }^{\circ} \mathrm{C}$ after added with $4 \mathrm{mg} / \mathrm{mL}$ IGP followed by four washes with PBS. The RBCs pellet was resuspended in PBS and sent for CLSM (Olympus-FV1000, Japan).

The cancer cell targeting potential of IGP-decorated liposomes was evaluated with confocal microscopy. Approximately $2 \times 10^{5}$ HEK293 or A549 tumour cells were grown on a 35-mm cell culture dish overnight and then incubated with $500 \mu \mathrm{l}$ IGP-decorated liposomes in $500 \mu \mathrm{l}$ fresh medium for $30 \mathrm{~min}$. Thereafter, the cells were carefully washed three times with PBS and fixed with $4 \%$ formaldehyde before sent for CLSM.

About $10^{6}$ A549 cancer cells were added with PBS or IGP decorated liposome at $2 \mathrm{mg} / \mathrm{mL}$ DOPC/DOPG and then incubated for $0.5-2 \mathrm{~h}$ followed by thrice washes with PBS. The cells were retrieved by centrifugation and resuspended in PBS at a cell density of $10^{5}-10^{6}$ cells $/ \mathrm{mL}$. The resuspension was loaded to a flow cytometry (Becton Dickinson, San Jose, CA, USA) to measure the GFP fluorescence.

\section{Sample preparation for electron microscopy}

E. coli cells treated with $4 \mathrm{mg} / \mathrm{mL}$ IGP fusion proteins were collected and processed by prefixation with $2.5 \%$ glutaraldehyde in $0.2 \mathrm{M}$ phosphate buffer $(\mathrm{pH}$ 7.3). Post-fixation, the samples were treated with $0.5 \%$ osmium tetroxide in Millonig's constant osmolarity phosphate buffer ( $\mathrm{pH} 7.4$ ), followed by dehydration in graded ethanol solutions. The dehydrated cell pellet was embedded in medium Taab Resin and polymerized for $24 \mathrm{~h}$ at $60^{\circ} \mathrm{C}$. Slices $(50 \mathrm{~nm})$ were prepared and stained with aqueous uranyl acetate and lead citrate. Stained slices were examined by TEM.

Freshly prepared DOPC:DOPG liposomes with or without IGP protein decoration were dropped onto a carbon-coated copper grid. The grid was negatively stained with $2 \%$ phosphotungstic acid and then rinsed with PBS. The grid was air-dried at room temperature (RT) and sent for TEM imaging. 


\section{In vivo near-infrared-fluorescence (NIRF) imaging study}

Animal experiments were carried out according to protocols approved by the Animal Care and Use Committee of Southeast University, China. Female BALB/C nude mice (4 6 weeks old) were reared in an Animal Environmental Control Unit. Approximately $2 \times 10^{6}$ A549 cells were subcutaneously inoculated into the right foreleg of each nude mouse. When the tumour reached over $0.4 \mathrm{~cm}$ in diameter (10-14 days after implantation), the mice were subjected to in vivo near-infrared fluorescence imaging studies.

The mice were weighed and grouped $(n=3)$ to control for weight differences. Preliminary experiments determined that doses below $1 \mathrm{nmol}$ Cy5/mouse gave weak signals. Therefore, Cy5RBC-Lip/Cy5 and Cy5-IGP-RBC-Lip were given at a dose of $25 \mathrm{nmol} \mathrm{Cy5/mouse} \mathrm{via} \mathrm{tail} \mathrm{vein}$ injection. The spectral fluorescence images consisting of autofluorescence and Cy5 spectra were obtained and then analysed based on their spectral patterns using Living Image 4.5.

\section{Data Processing and Statistics}

The experiments were independently repeated (repeats $\geq 3$ ) and the representative results were plotted. Unless stated otherwise, all the results are expressed as mean \pm standard deviation (SD). One-way analysis of variance (ANOVA) and student's t-test were performed to compare the difference between groups. 


\section{Tables}

Table S1. Protein samples used in this study

\begin{tabular}{lllllc}
\hline Proteins & N-terminal & C-terminal & MW & pI & $\begin{array}{c}\text { Yield } \\
\text { culture) }^{\mathbf{a}}\end{array}$ \\
\hline Ib-AMP4 & QWGRRCCGWGP & $/$ & 2,543 & 10.70 & $/$ \\
& GRRYCRRWC & & & \\
Thanatin & GSKKPVPIIYCNR & $/$ & 2,291 & 10.48 & $/$ \\
& RSGKCQR & & & \\
P1c & RGDGSIRTPKISK & $/$ & 2,142 & 10.28 & $/$ \\
& PIKFEL & & & \\
Ib-AMP4-GFP-P1c & MQWGRRCCGW & HHHHHHGGGSG & 35,050 & 6.65 & \\
(IGP) & GPGRRYCRRWC & GGSGGGSGIRTP & & & \\
& GGGSGGGSGGGS & KISKPIKFELSGS & & & \\
& GSGSG & GRGD & & & \\
\hline
\end{tabular}

$\overline{{ }^{a} \text { Protein expression was not well optimized, and the yield was calculated as the amount of purified }}$ soluble fusion protein obtained from each liter of cell culture. GFP, green fluorescence protein; MW, molecular weight; IP, isoelectric point. 


\section{Figures}
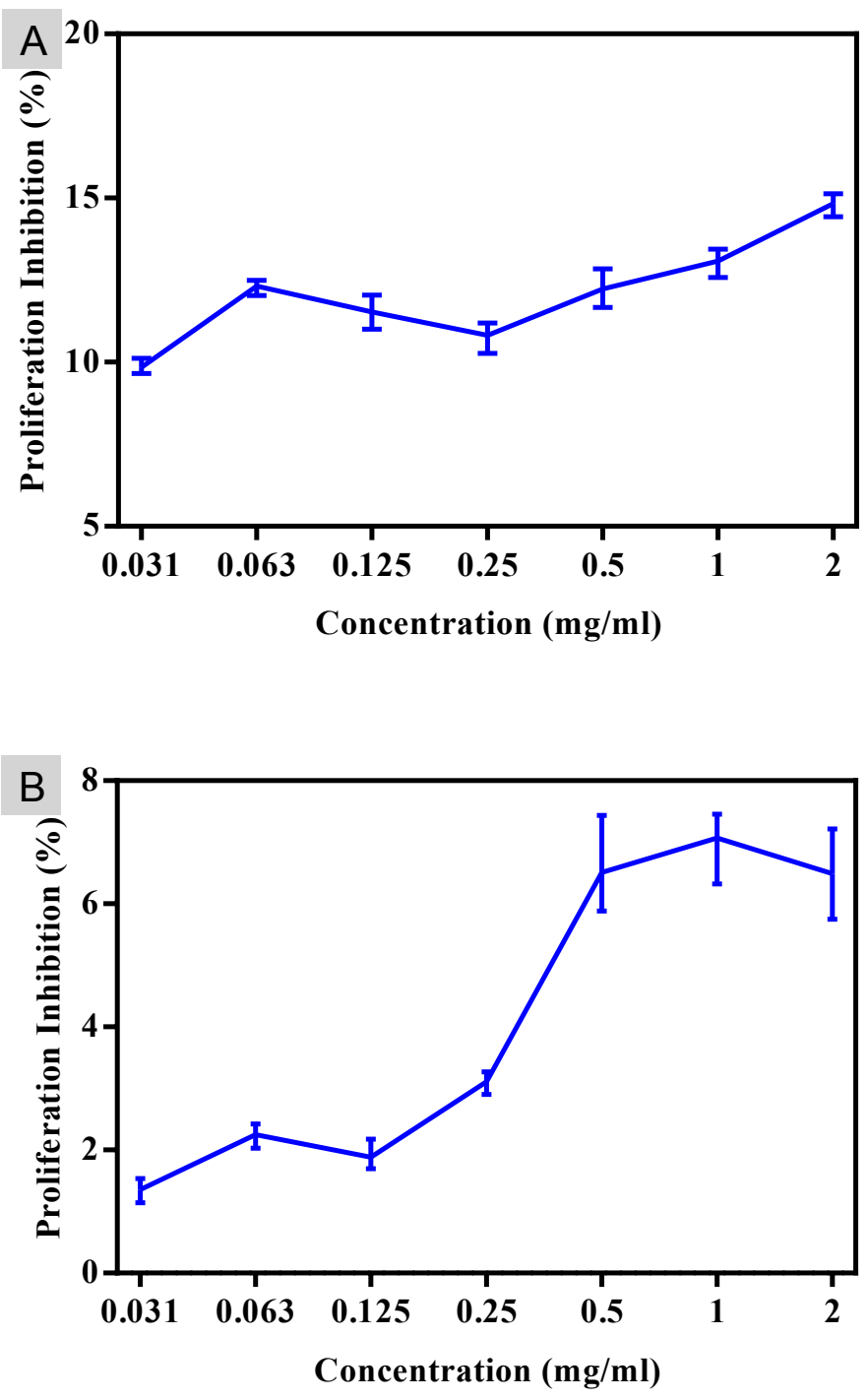

Figure S1. The cytotoxicity of the purified fusion proteins. The cytotoxicity of the purified proteins was evaluated in A549 (A) and HEK293 (B) cells by MTT assay. About 5,000 cells were seeded in each well and grown until the cell density reached about $30 \%$. Different concentrations of the proteins were then added and continuously cultured for $24 \mathrm{~h}$. Afterward, $0.5 \%$ MTT was added and the cells were cultured for another $4 \mathrm{~h}$. The media were then discarded and $150 \mu \mathrm{DMSO}$ was added to each well before sent to OD560 measurement. PBS was used as negative control defined as $0 \%$ inhibition. Each measurement was performed for three times and the results were presented as the mean value with standard deviation. 


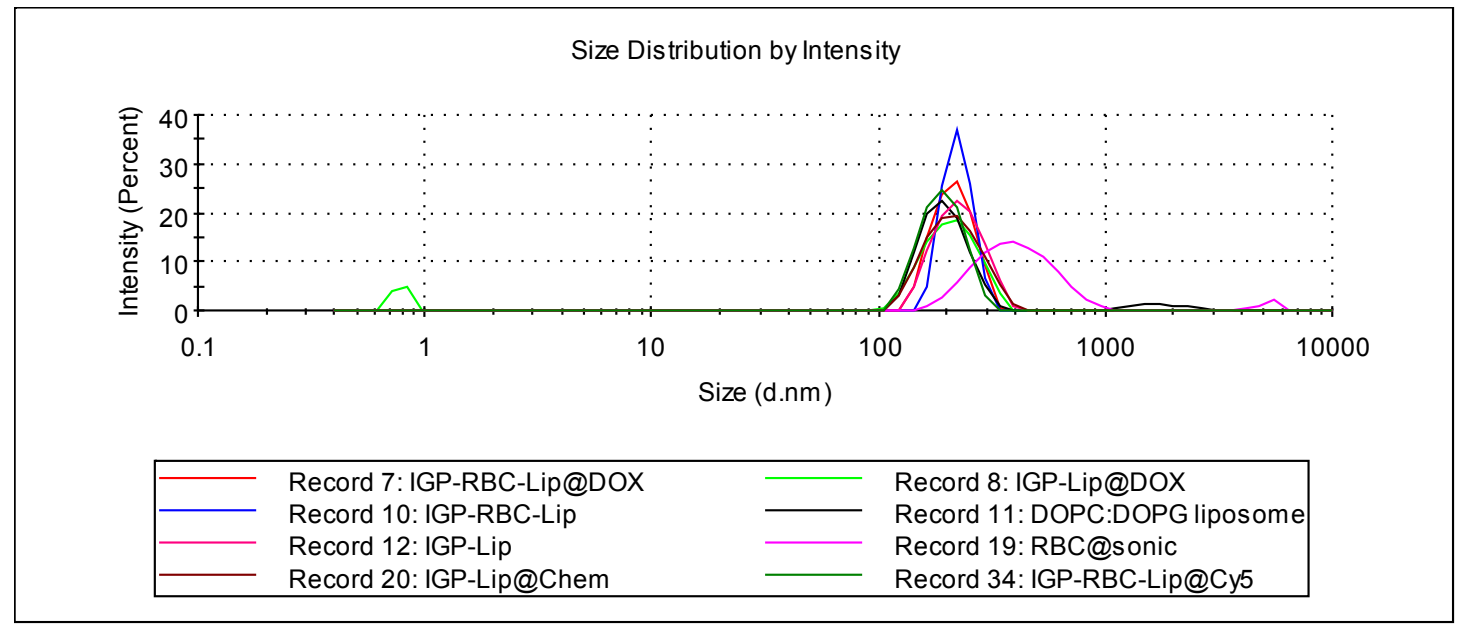

Figure S2. The size distribution of the prepared liposomes. The RBCs after decorated by IGP was firstly treated with sonication and the particle size was between 200 to $1000 \mathrm{~nm}$ in diameter. The liposomes prepared by extrusion method were $\sim 200 \mathrm{~nm}$ in diameter. 


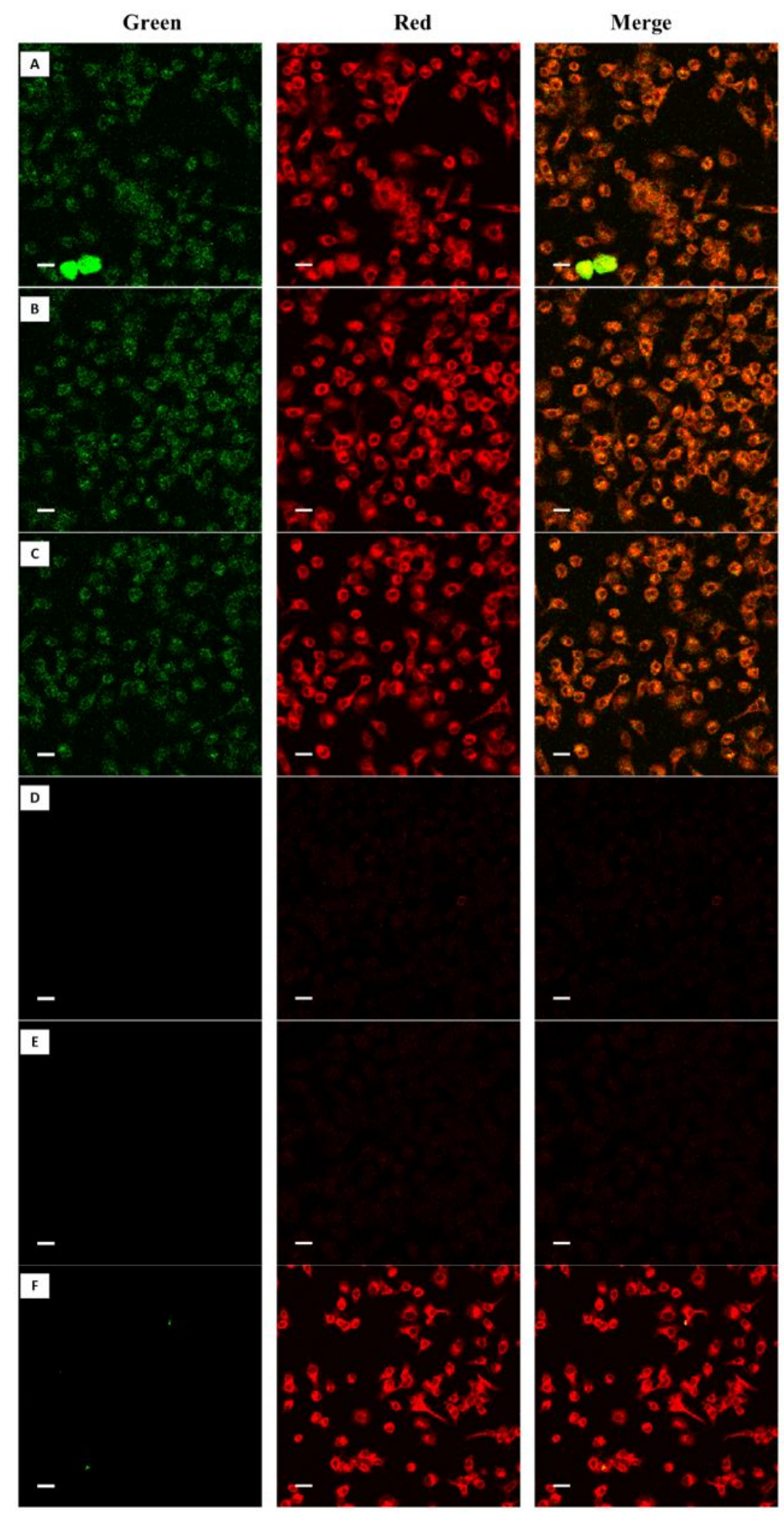

Figure S3. IGP decorated liposomes concentrate in A549 cells and increase the cellular DOX accumulation. Cells seeded in confocal microscope glasses were treated with IGP-Lip-DOX (A), IGP-Lip-DOX@Chem (B), IGP-RBC-Lip-DOX (C), P1c+IGP-Lip-DOX (D), Lip-DOX (E ) or DOX (F), separately, followed by incubation of $30 \mathrm{~min}$. Scale bar $20 \mu \mathrm{m}$. 


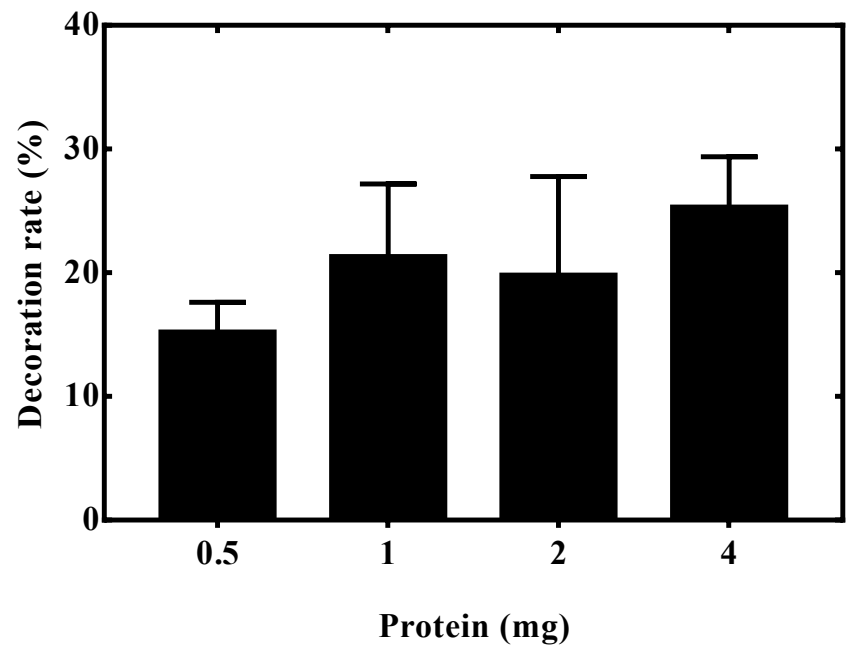

Figure S4. IGP proteins decorating RBCs at different concentrations. The cell pellet of approximately $10^{9} \mathrm{RBCs}$ was lysed in $\mathrm{ddH}_{2} \mathrm{O}$. Lysis-resuspension cycles were repeated until the pellets became white. The cell pellets were mixed with $0.25,0.5,1$ and $2 \mathrm{ml}$ IGP solutions (2 $\mathrm{mg} / \mathrm{ml}$ ), separately. After incubation for 10-60 min, the supernatant was retrieved after centrifugation at 1,0000 rpm for $20 \mathrm{~min}$, and the IGP protein concentration recovered from the supernatant was determined by measuring the green fluorescent intensity. The decoration rate was calculated using the following equation: decoration rate $(\%)=\left(C_{0}-C\right) / C_{0} \times 100 . C_{0}$ is the IGP concentration before added into the cell pellets, and $C$ is the IGP concentration recovered from the supernatant after decoration. Each measurement was performed for three times and the results were presented as the mean value with standard deviation. The results are representative of replicate experiments. 


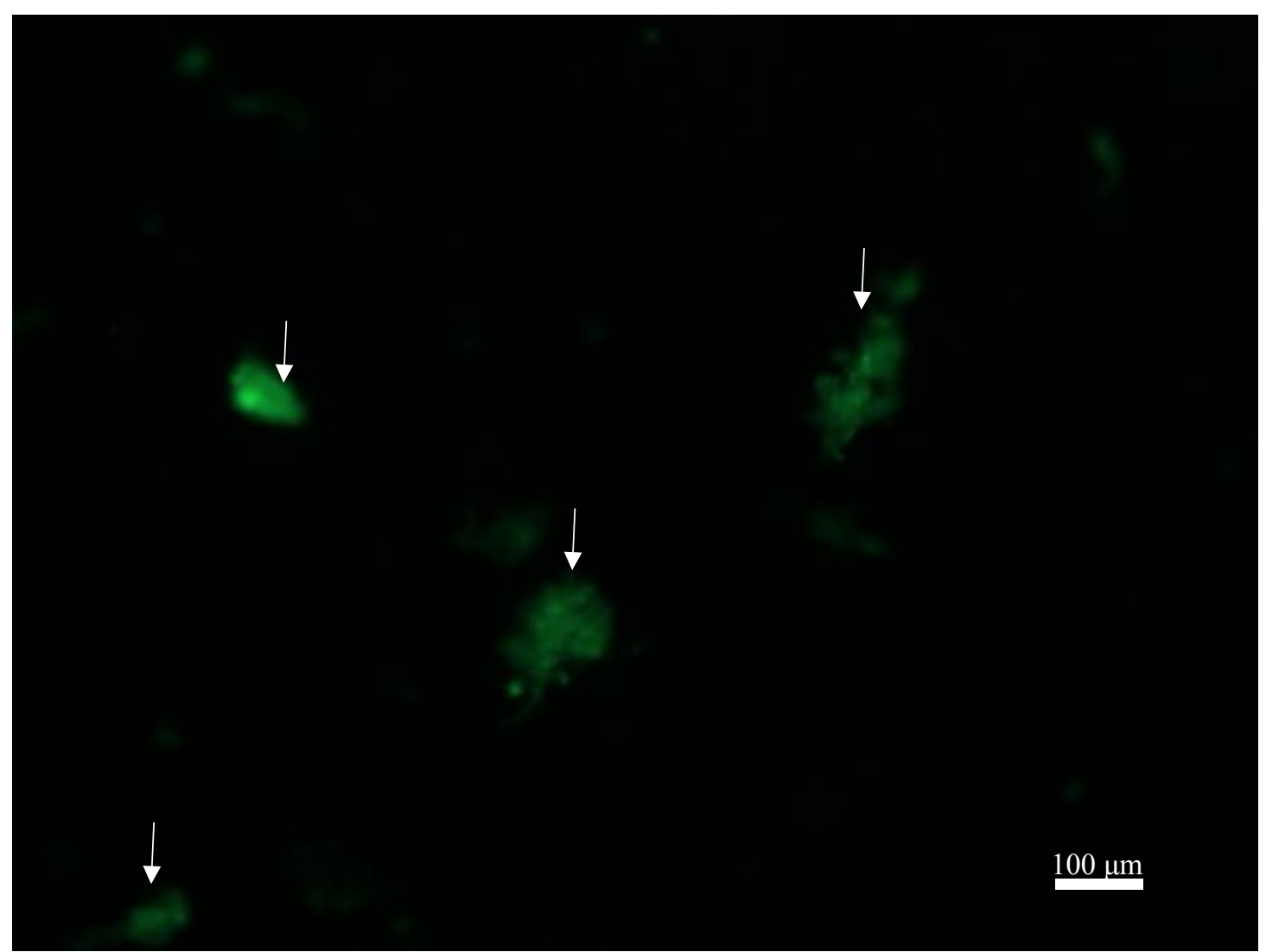

Figure S5. IGP proteins decorate DOPC-DOPG membrane debris. The DOPC-DOPG

liposomes were prepared by thin film evaporation method. After the thin film was prepared, IGP in 5\% glucose was added to rehydrate the lipid film. The membrane debris was stained in green fluorescence under microscope after washed trice with PBS. The arrow indicated DOPC-DOPG membrane debris. 


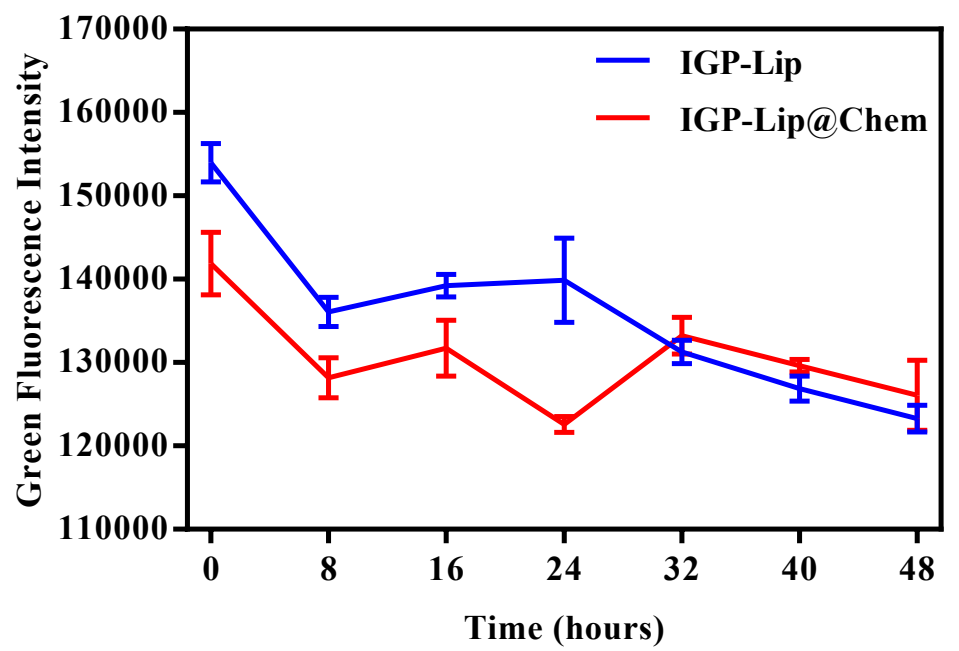

Figure S6. The green fluorescence intensity of the IGP decorated liposomes prepared by two methods. The IGP decorated liposomes prepared by two methods were supplemented with $10 \%$ bovine serum and then dialyzed (MW cut off $300 \mathrm{kD}$ ) against PBS containing $10 \%$ bovine serum. An aliquot was retrieved at each interval and measured with green fluorescent intensity. A very limited fluorescence loss below 15\% was observed for both preparations, but it could be accounted to the volume change due to water transport across the dialysis membrane. Each measurement was performed for three times and the results were presented as the mean value with standard deviation. 


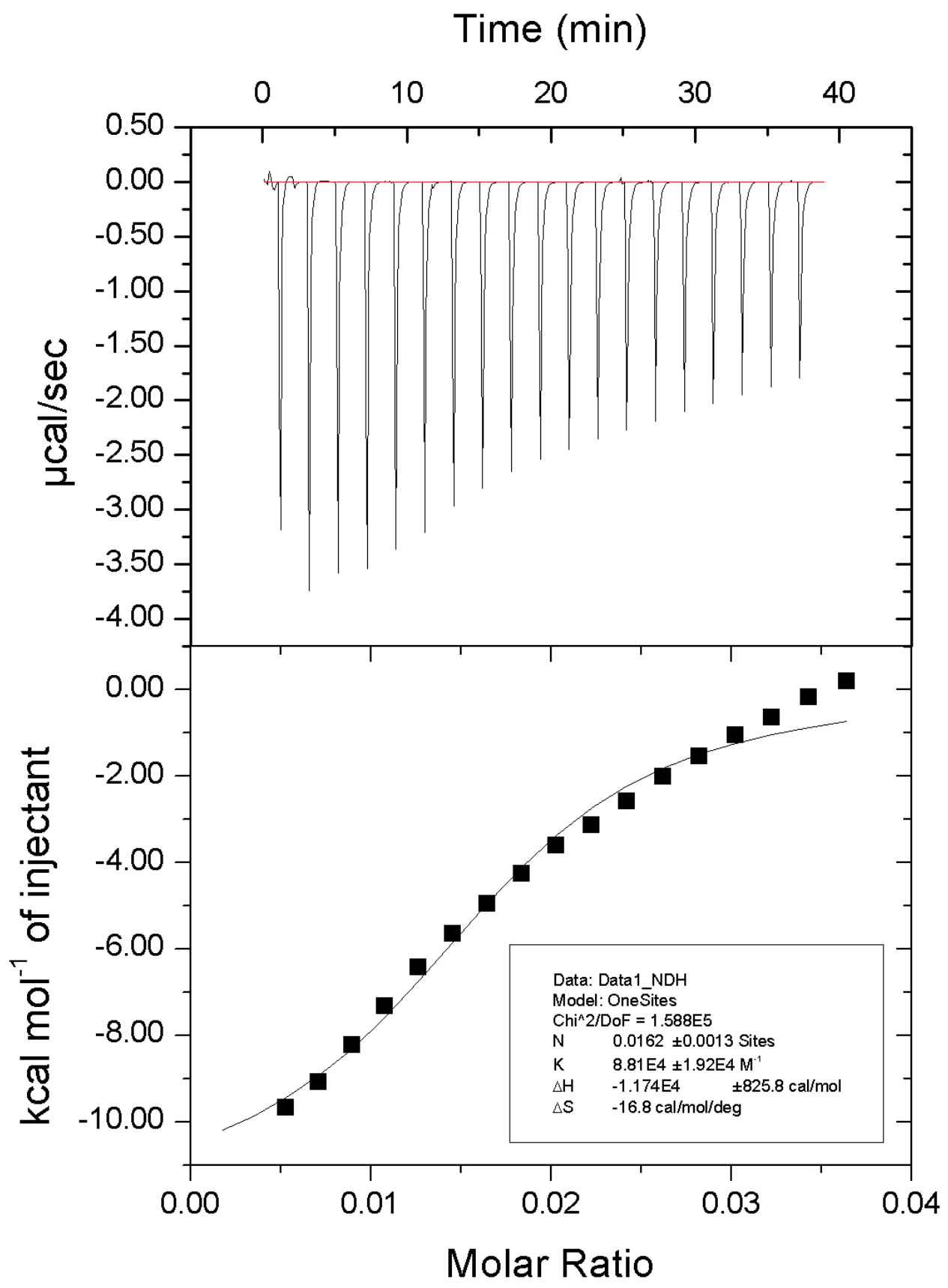

Figure S7. The thermodynamics of PGT binding lipid bilayer by ITC in PBS. In comparison with saltfree glucose, the enthalpy $(\Delta H)$ produced by each aliquot injection was much greater in PBS. The binding was saturated in lower molar ratio and occurred more easily in salt-free conditions $(K)$. 


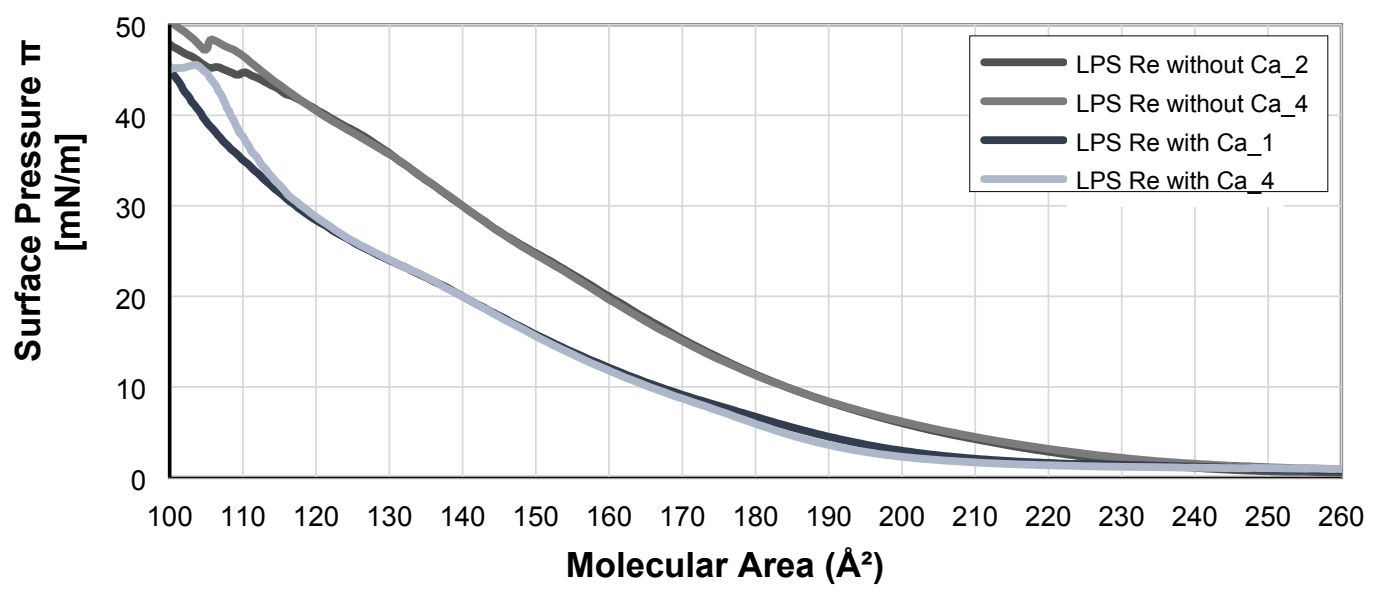

B

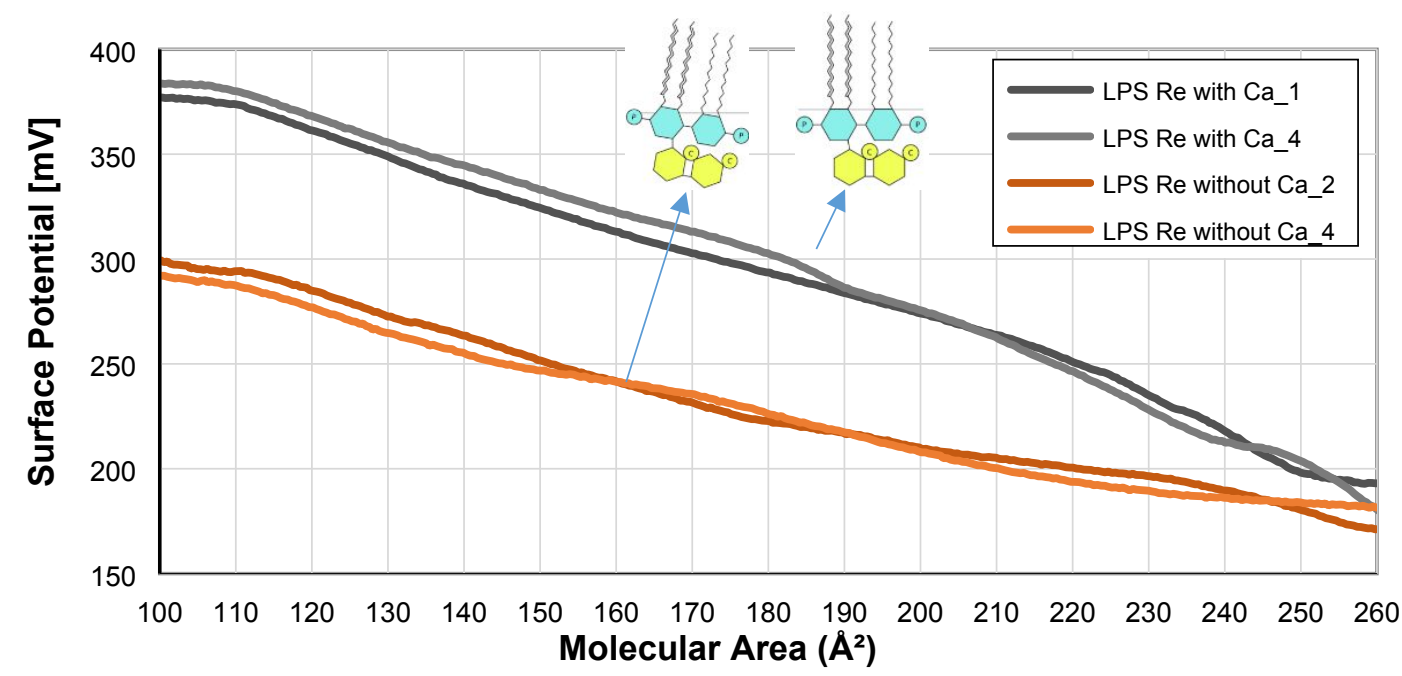

Figure S8. Surface area isotherms for lipopolysaccharide (LPS-Re). A) Surface pressure isotherms for the LPS-Re monolayer in calcium-loaded and -free buffers. In the $\pi$-A isotherms for LPS-Re, we observed the effect of calcium ions on condensation in the following data: (i) the onset of pressure increase appeared at the lower molecular area and (ii) the surface pressure was always lower than that in the calcium-free subphase in the same molecular area. These findings indicated that calcium affects the intermolecular interactions between LPS molecules when the headgroups were compact. B) LPS-Re Surface Potential isotherms for the LPS-Re monolayer in calcium-loaded and -free buffers. Calcium increases the effective molecular moment. The presence of $\mathrm{Ca} 2+$ leads to a higher normal moment indicating that the hydrocarbon chains of LPSRe directed more perpendicular to the surface. 
A

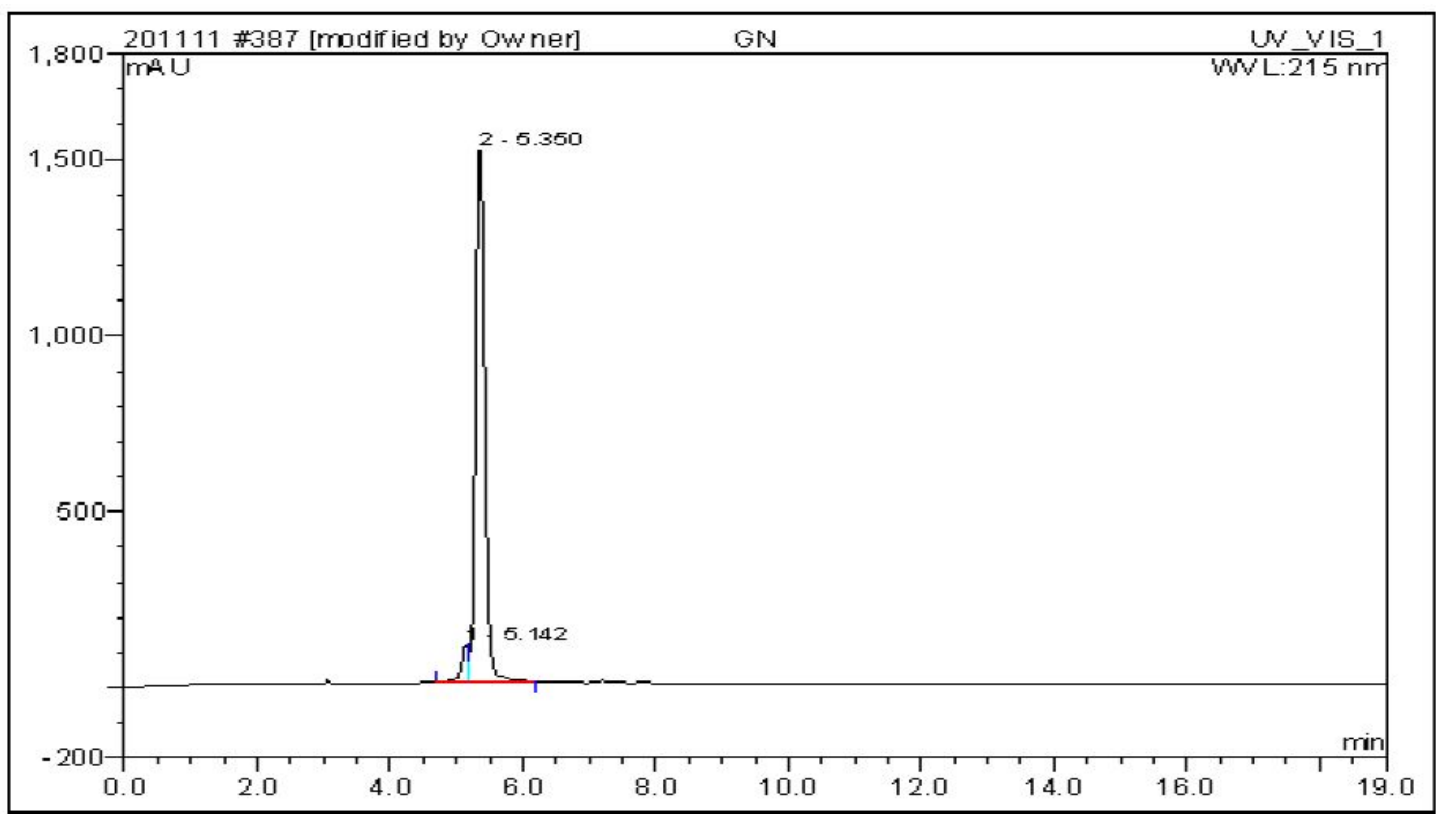

B

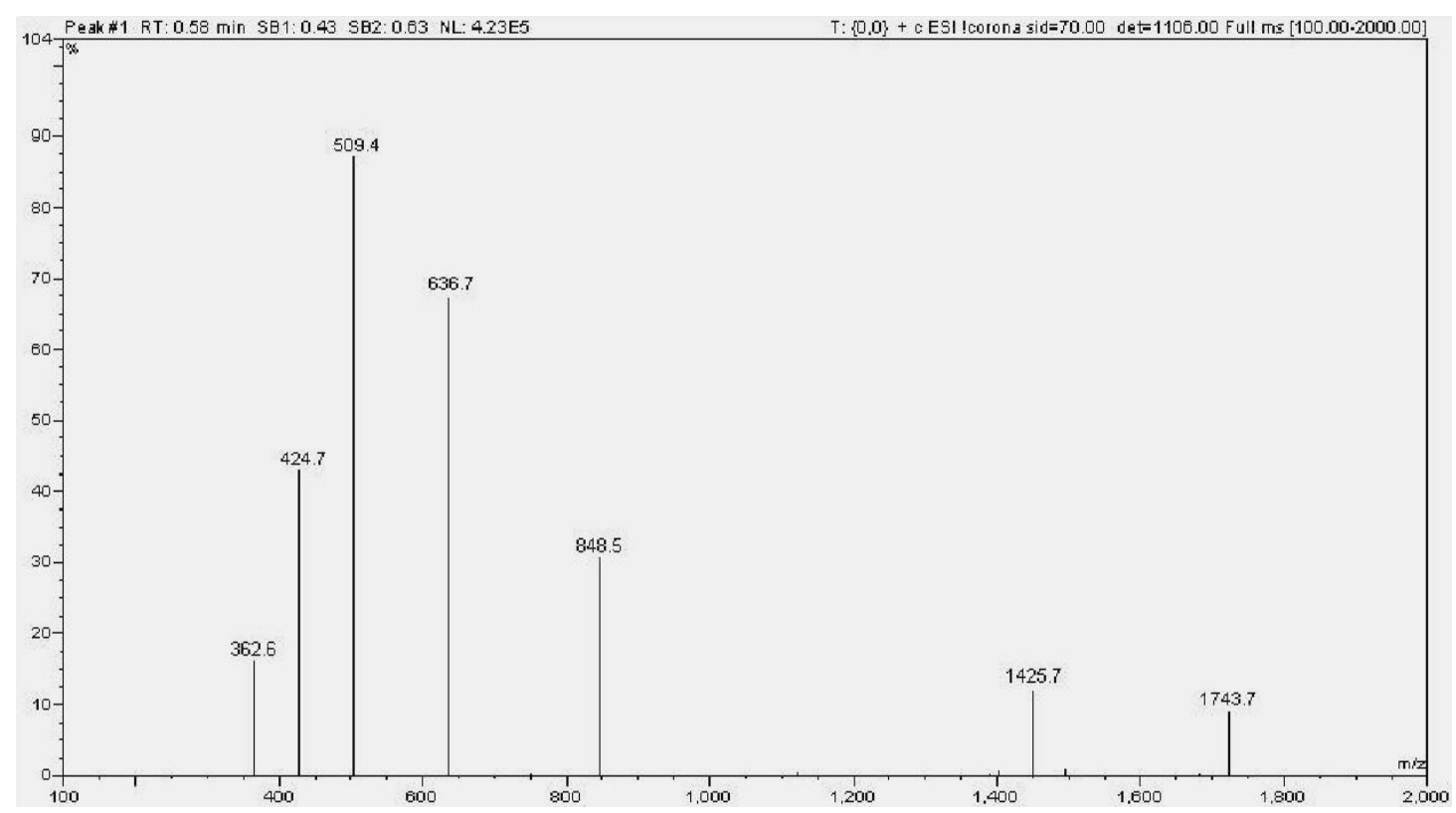

C 


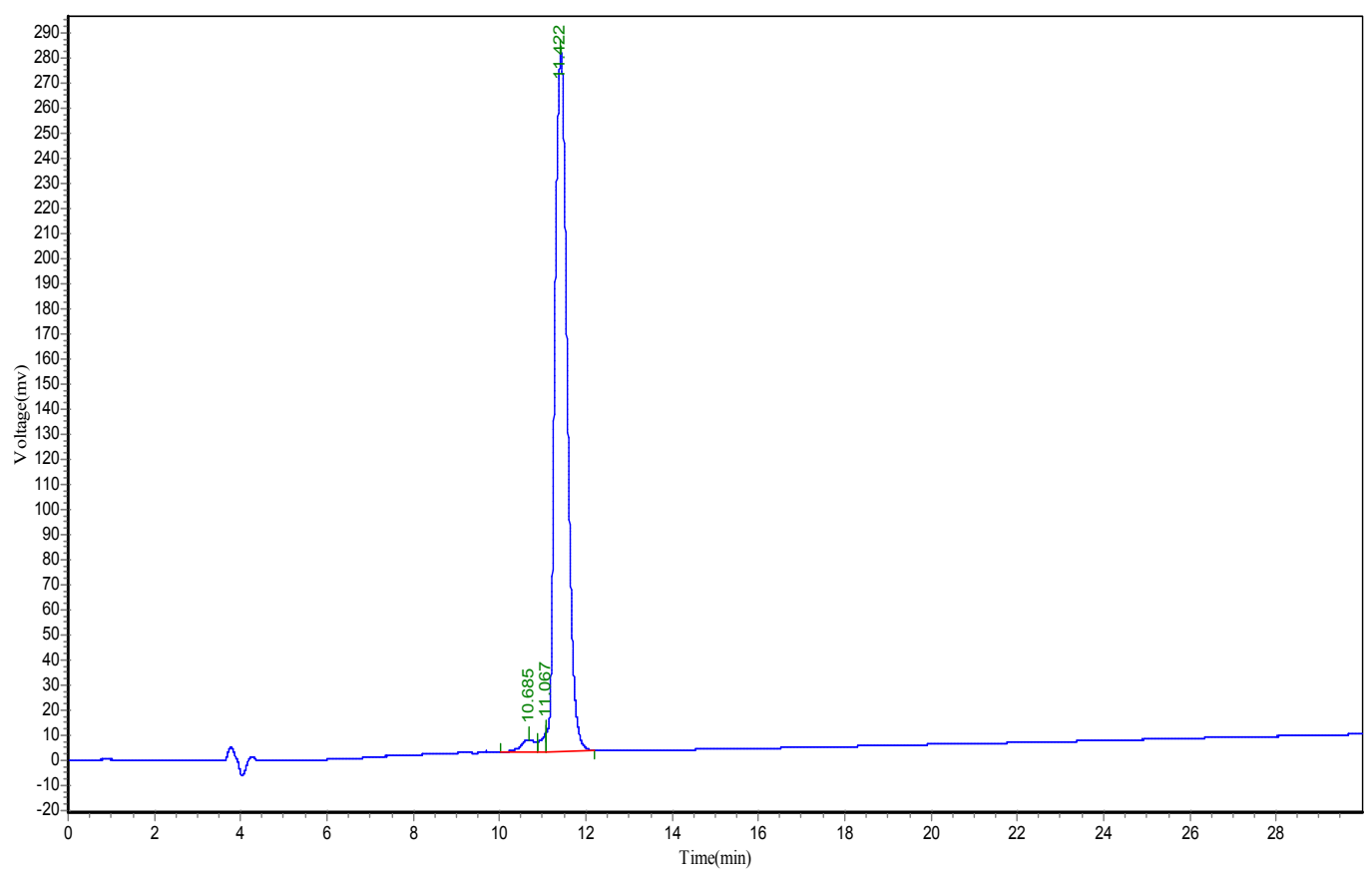

D

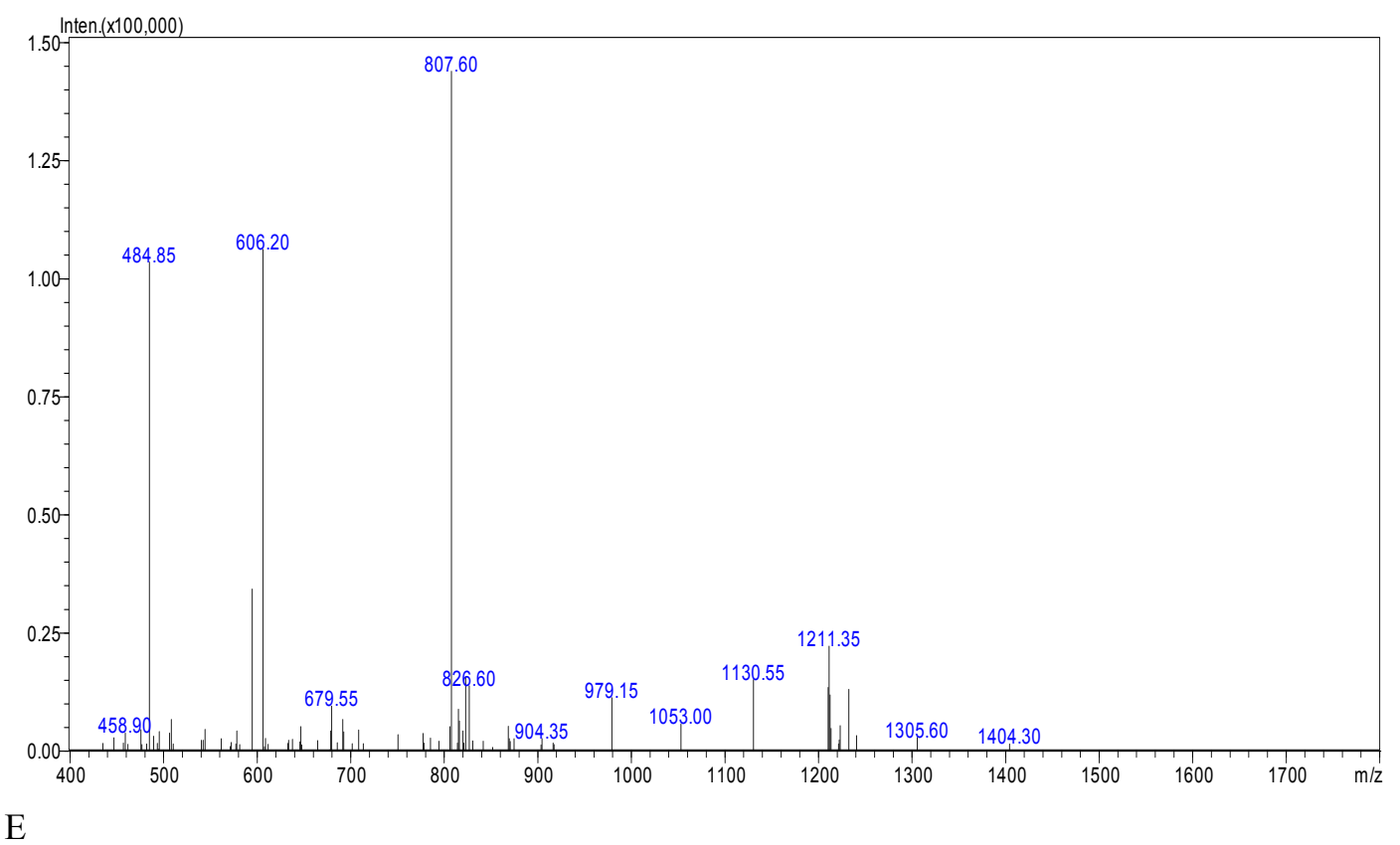




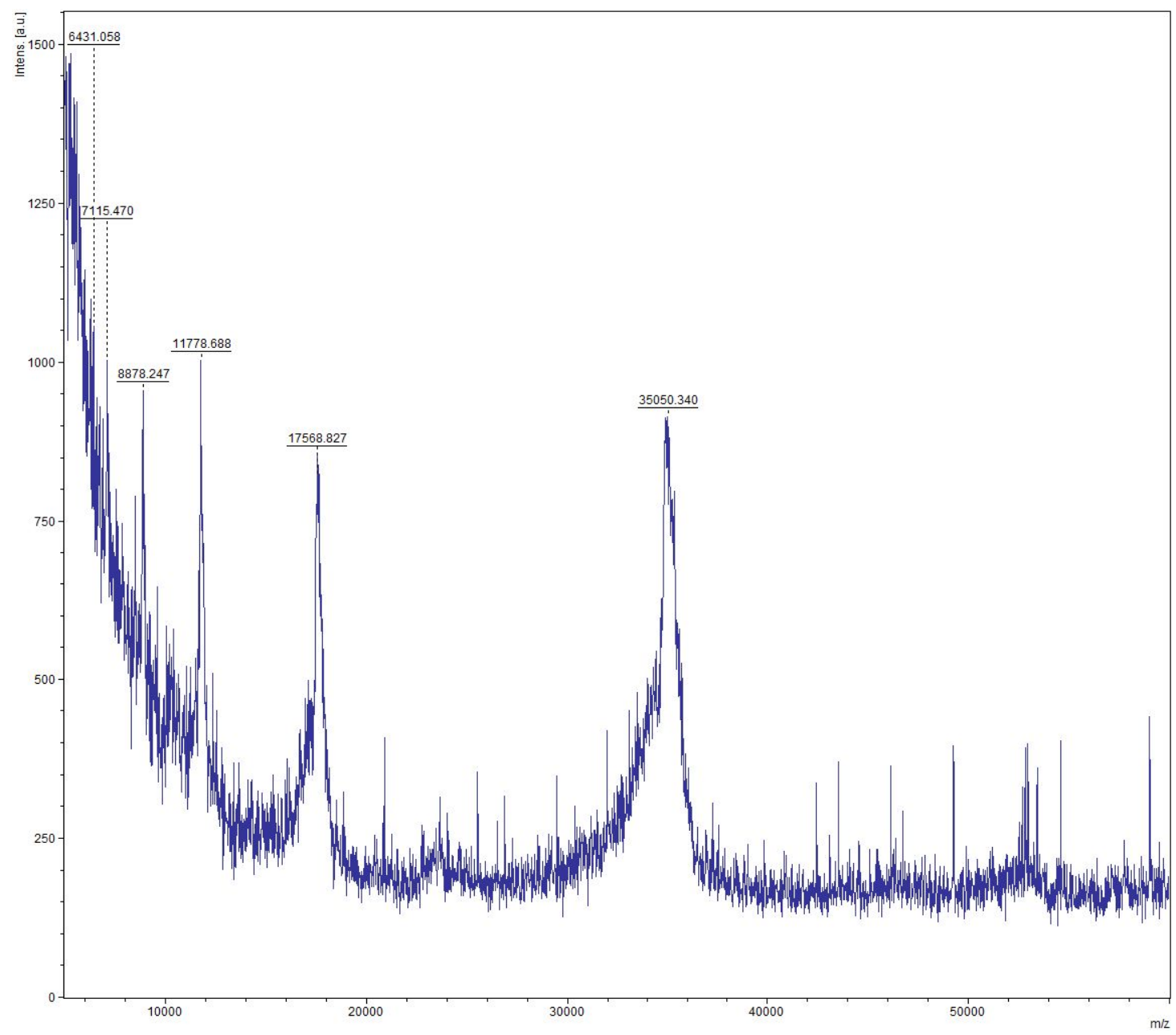

Figure S9. The HPLC and Mass spectrum of the peptides. HPLC (A)and (B) Mass spectrum for Ib-AMP4; HPLC (C) and Mass spectrum (D) for P1c; Mass spectrum for IGP (E).

\section{Reference}

(1) Fan, X., Reichling, J., and Wink, M. (2013) Antibacterial activity of the recombinant antimicrobial peptide Ib-AMP4 from Impatiens balsamina and its synergy with other antimicrobial agents against drug resistant bacteria. Pharmazie 68, 628-30.

(2) Oliveira, S., Schiffelers, R. M., van der Veeken, J., van der Meel, R., Vongpromek, R., van Bergen En Henegouwen, P. M., Storm, G., and Roovers, R. C. (2010) Downregulation of EGFR by a novel multivalent nanobody-liposome platform. J. Controlled Release 145, 165-75. 